\title{
Degradation processes in LED modules
}

\author{
V.M. Sorokin ${ }^{1}$, Ya.Ya. Kudryk ${ }^{1}$, V.V. Shynkarenko ${ }^{1}$, R.Ya. Kudryk ${ }^{2}$, P.O. Sai ${ }^{1}$ \\ ${ }^{I}$ V. Lashkaryov Institute of Semiconductor Physics, NAS of Ukraine \\ 41, prospect Nauky, 03680 Kyiv, Ukraine, \\ E-mail:kudryk@isp.kiev.ua; shynkarenko@isp.kiev.ua \\ ${ }^{2}$ Ivan Franko National University of Lviv \\ 1, Universytetska str., 79000 Lviv, Ukraine
}

\begin{abstract}
Electrical-heat-light degradation model of a light-emitting module has been developed in this work. The Monte-Carlo method was used to calculate the reliability time of LED modules with different halfwidth of LED chip series resistance. Separation of LED chips with different series resistance before assembling may increase the time of emission in a stable mode up to $10 \%$.
\end{abstract}

Keywords: LED, degradation, thermal resistance, junction-to-heatsink thermal resistance.

Manuscript received 16.03.16; revised version received 27.07.16; accepted for publication 13.09.16; published online 04.10.16.

\section{Introduction}

Evaluation of the reliability time is a necessary stage of device development and electronic engineering. If one connects several microelectronic devices in single system, their total reliability, as will be shown below, will depend not only on the reliability of the initial components, but also on a number of other process parameters. Excess reliability of the device is as harmful to the company as the insufficient one, because of imposing financial losses [1,2]. For example, the LED module with excess reliability may be used in the mode of enhanced output power, but it lowers its reliability, which will increase competitiveness of the LED modules on the market.

The series resistance variation of LED chips in the module results in increase of variance parameters inherent to LED modules and reduce their reliability. In the case of parallel connection of LED modules this issue becomes especially important. Additional LED chip binning can reduce the variance of parameters of chips and increase reliability of the resulted LED module. On the other hand, additional LED chip binning enhances the cost of equipment operation and personnel work. To assess feasibility of this process, it is necessary to model the effect of spread parameters of LED chips on a LED module luminous flux and take into account the influence of degradation of these LED chip parameters.

\section{Methods and samples}

We tried to analyze operation mechanisms of ohmic contacts to $\mathrm{GaN}$ in modern light emitting devices. As samples, we took LED modules produced by national industry and based on LED chips from "EDISON Optocorporation" before luminophor filling. Two modules with identical manufactory processes were 
explored. They were manufactured on $\mathrm{PCB}$ with $\mathrm{Al}$ cooler at the back and LED chips on top. Chips were connected at series-parallel circuit, shown in Fig. 1. Characteristics of LED module are as follows: maximum current $0.35 \mathrm{~A}$, maximum voltage $24 \mathrm{~V}$, maximum dissipation power $8 \mathrm{~W}$.

In the first module was cut all connections between chips for learning of individual characteristics of real chips after influence of PCB soldering and similar manufacture processes. The second module was turned on for a long-term observation at a stabilized temperature of heat sink.

To formulate LED module degradation model, we build electrical-heat-light model of LED chips, which was based on experimental $I-V$ curves and their variation. Then, we calculated heat variations between chips and predicted each LED chip degradation and, as a result, calculated LED module degradation and change in its emission. Let's combine some groups of LED matrix with different variations of LED chips series resistance and find a best result in emission time, if we use additional sorting the LED chips.

\section{Current transfer mechanisms in a chip. $I-V$ model}

I- $V$ characteristics and $C$ - $V$ characteristics were used for research of current transfer mechanisms in each LED chip. Main parameters of LED chips were found for the following modelling. As the maximum current for a
LED module was $0.35 \mathrm{~A}$, we chose current at $I-V$ measurements within the range of $10^{-11} \ldots 10^{-1} \mathrm{~A}$. In Fig. 2, it is shown the forward bias of $I-V$ characteristic.

As one can see, inside the LED module there were three chips with high parasitic currents (group I, in Fig. 2). These currents may belong to a defect that may lead to a quick chip degradation. Also, one can see two chips with a high value of series resistance (group II in Fig. 2). The current will flow through these chips 3 times lower than through other chips, as a result, neighbor chips parallel to it will be overloaded, and, consequently, will degrade quicker.

We calculated mean and variance chip characteristic from $I-V$ and $C-V$ curves and use it in modelling the LED module dependence on defects of different types. $I-V$ characteristics of one chip within the temperature range $290 \ldots 370 \mathrm{~K}$ were measured to ascertain the current transfer mechanism and LED chip parameters of exploration.

$I-V$ characteristic of a LED chip within the range $290 \ldots 370 \mathrm{~K}$ are shown in Fig. 3a. We found three characteristic areas. The exponential areas I and II are related with the processes in $p-n$ junction. The approximately linear area III (Fig. 3b) is related with current flow processes both in bulk $p$ - and $n$-regions and in semiconductor-metal ohmic contacts. Some nonlinear properties in the area III, as will be shown below, are related with Joule heating the $p-n$ junction and bulk of the chip.

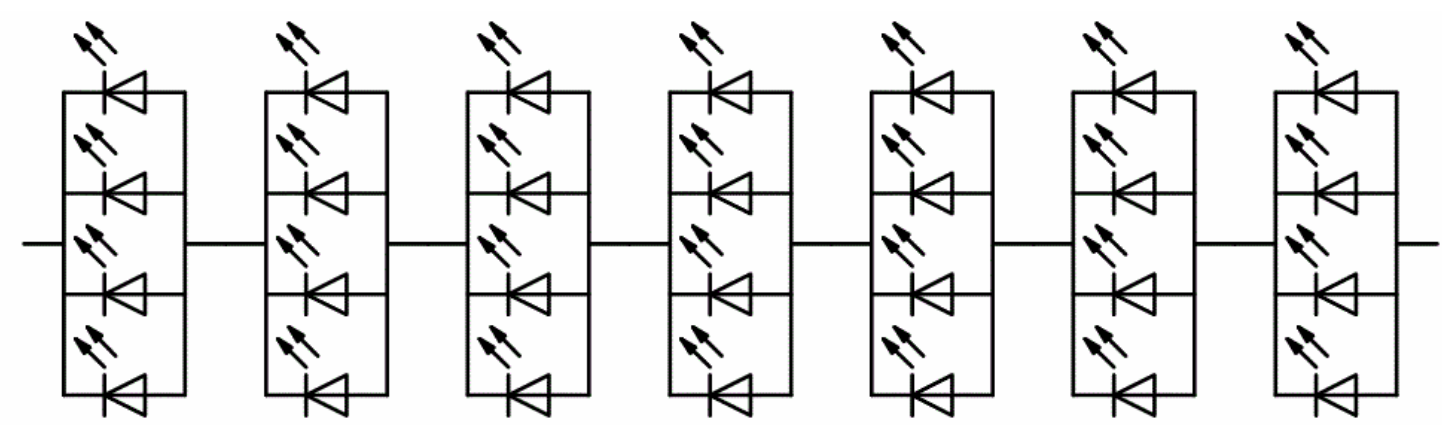

Fig. 1. Series-parallel circuit of LED chips connection in LED module.
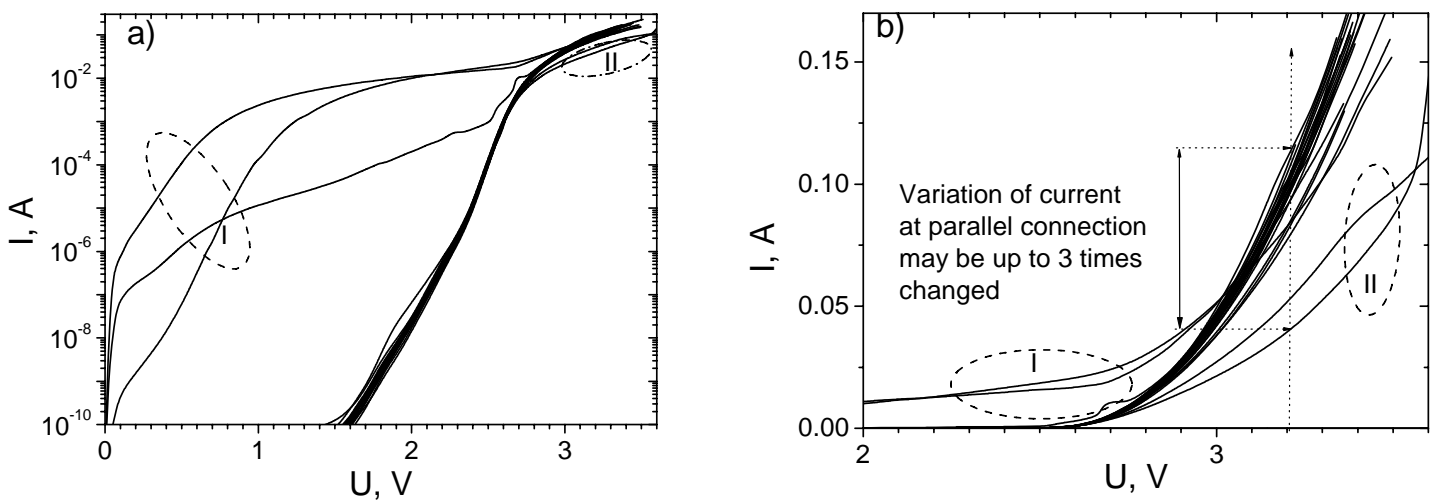

Fig. 2. Forward bias of LED chip $I-V$ characteristic in half-logarithmic (a) and linear (b) scales. 

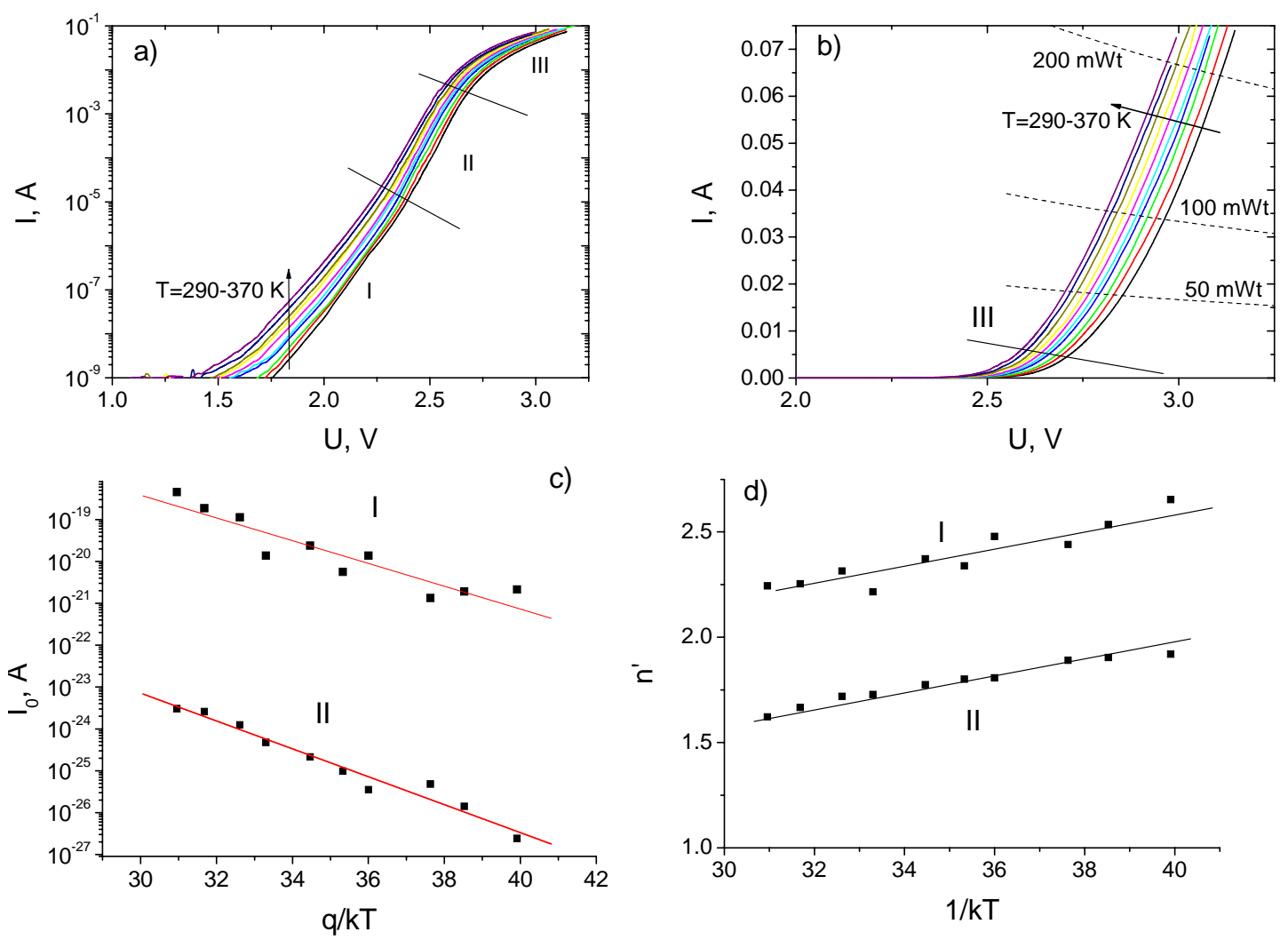

Fig. 3. Temperature dependences of LED chip $I-V$ characteristic in half-logarithmic (a) and linear (b) scales and the temperature dependences of calculated saturation current (c) and quality factor (d) in areas I and II.

The first two areas can be described by Shockley diode equation [3]:

$$
I=I_{0}\left(\exp \left(\frac{q V}{n k T}\right)-1\right) \text {. }
$$

The temperature dependence of the saturation current $I_{0}$ is well described by the following expression (Fig. 3c):

$$
I_{0}=I_{0 i} \exp \left(-\frac{E_{b}}{k T}\right) .
$$

The temperature dependence of ideality factor may be related with series connection of contacts with quantum-mechanical tunneling (or thermoionic emission) current mechanism. In this case, the voltage drop in a contact leads to additional changes in the calculated value of ideality factor. The total voltage drop on the LED chip is the sum of those on the $p$ - $n$ junction and contacts:

$$
V=\ln \left(I / I_{0}\right) n k T / q+\ln \left(I / I_{0 F}\right) E_{00} / q,
$$

So, when one deduces the ideality factor as:

$$
n^{\prime}=q / k T d \ln (I) / d V \text {, }
$$

he receives:

$$
d V=d \ln (I)^{n k T} / q+d \ln (I)^{E_{00}} / q,
$$

as a result:

$n^{\prime}=n+E_{00} / k T$

As one can see in Fig. 3d, the temperature dependence of ideality factor on both areas is well described by the dependence like (6) with the same Padovani-Stratton parameter $\left(E_{00}\right)$ [3]. It may indicate a similar origin of the temperature dependence of quality factor in areas I and II of $I-V$ curves. The PadovaniStratton parameter derived from calculations of the temperature dependence of quality factor is close to $k T$. It indicates the possibility of current flow caused by thermoionic emission.

Considering the area III of $I-V$ curves, one should take into account possible additional heating by the current. Temperatures of LED chip $p-n$ junction, region of spreading current and ohmic contacts are assumed to be almost identical (Fig. 4). Since the subwafer of LED chip is thicker more than an order as compared to the active layer, then additional heating equals:

$\Delta T=R_{t} P=R_{t} V I$, 
where $R_{t}$ is the junction-to-heatsink thermal resistance for each chip. In this case, our model will not take in account the temperature dependence of spreading length in the spreading layer and other heat processes that can lead to the temperature dependence of $R_{t}$.

If one sketches the lines with similar power dissipation (Fig. 3b), then their intersection with $I-V$ curves gives us points in which the magnitude of temperature changes due to current heating will be the same. As one can calculate the differential resistance in these points, it is simple to calculate the temperature dependence of this differential resistance at different dissipation powers (Fig. 5). It follows from Fig. 5 that, at scattering powers higher than $50 \mathrm{~mW}$, the temperature dependence of differential conductivity is kept constant. Thus, the main contribution to the series resistance is provided by the ohmic contact with the tunneling or thermoionic emission mechanisms.

If a significant contribution was due to the spreading current layer, it would lead to a falling temperature dependence of the semiconductor resistance. This conclusion is in good agreement with the temperature dependence of quality factor mentioned above. The results allow to simplify the model by ignoring the contribution of spreading current layer.
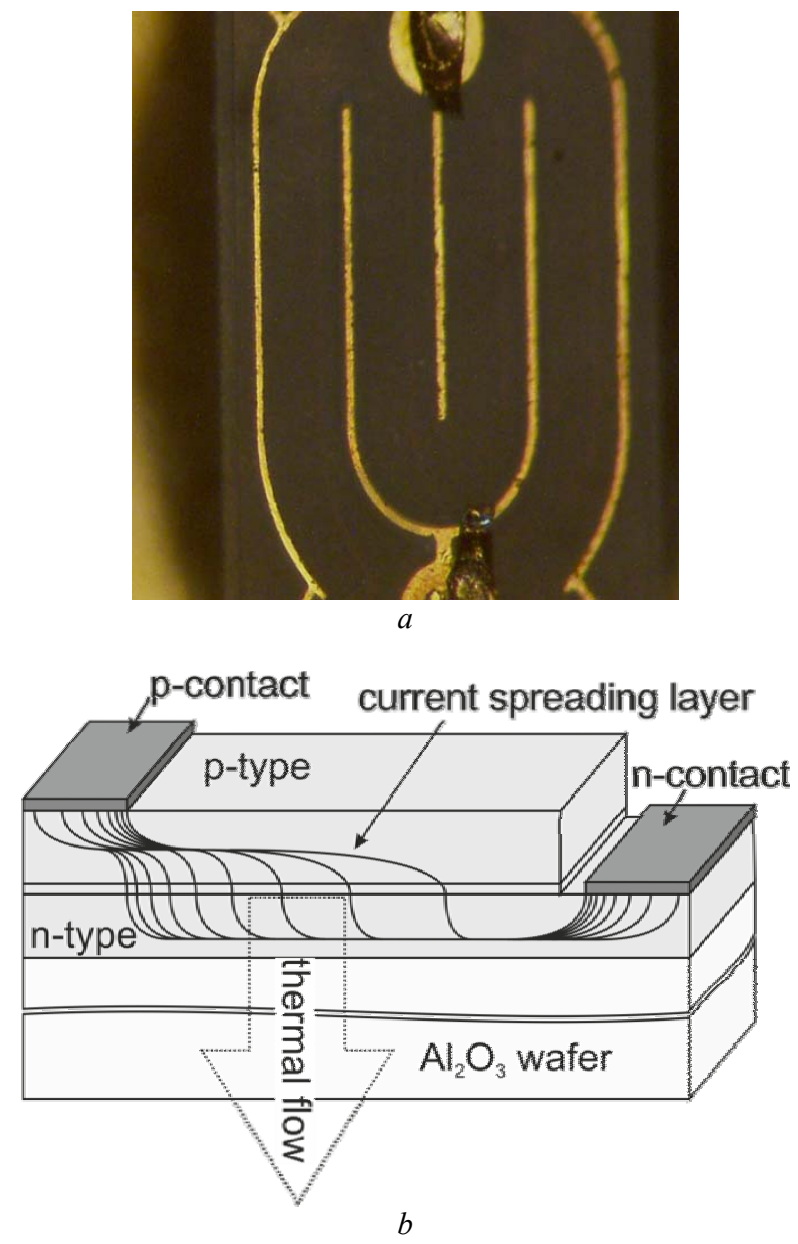

Fig. 4. LED chip (a) and the scheme of current flow in it (b).

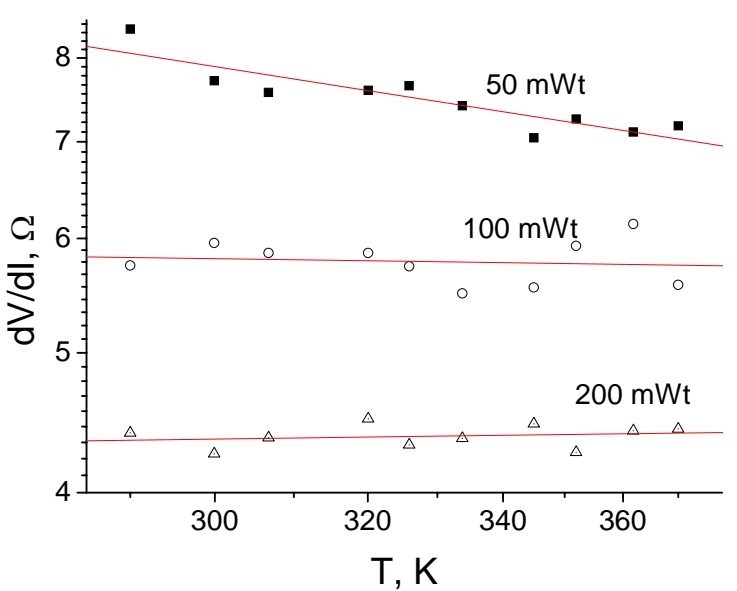

Fig. 5. Temperature dependence of differential resistance at different dissipation powers.

The differential resistance in the area III for the $I-V$ curve may be described using differentiation of Eq. (3). The term of the area $\mathrm{I}$ in $I-V$ characteristics can be neglected, since it minor influences at higher voltages. When taking (7) into account, one can obtain the following dependence:

$Q \equiv \frac{d V}{d I}=\frac{n k T}{q} \frac{1}{I}+\frac{E_{00}}{q} \frac{1}{I}=\frac{n k\left(T_{0}+R_{t} V I\right)+E_{00}}{q I}$,

Let us multiplicate both parts of equation (8) by current:

$Q I=\frac{n k T_{0}+E_{00}}{q}+\frac{n k}{q} R_{t} P$,

where $P=I V$.

Thus, one can draw dependence:

$\frac{d V}{d I} \frac{1}{V}=f\left(\frac{1}{P}\right)$

After linear approximation of this dependence in the area III $(I>0.03 \mathrm{~A})$, one can find cut-off equal to $\frac{n k}{q} R_{t}$ and calculate the thermal resistance value.

However, this value gives us the upper limit of thermal resistance due to influence of serial resistance of spreading current layer.

\subsection{Temperature of $p$ - $n$ junction at operating mode}

The averaged thermal resistance of LED chip on the heat sink can be found by examining the temperature dependence of the LED module efficiency, which is taken at different values of the stabilized current (Fig. 6). It is seen that increasing the integrated current by $10 \mathrm{~mA}$ leads to the same reduction in the efficiency like to heating at $34{ }^{\circ} \mathrm{C}$. In terms of a separate chip, it will give the thermal resistance value equal to $420 \mathrm{~K} / \mathrm{W}$. 


\subsection{LED matrix model}

Let's construct the model of integrated $I-V$ LED matrix consisting of serial connections of $w$ sections, each of which containing $u$ LED chips connected in parallel on a common heat sink. The voltage drop in individual LED chip that is placed in the section $j$ at the number $i$ and consists of the voltage drop in the $p$ - $n$ junction, in serial resistance of region with spreading current, and the drop voltage in ohmic contact:

$V_{j}=V_{p n, i j}\left(I_{i j}, T_{i j}\right)+V_{R, i j}\left(I_{i j} T_{i j}\right)+V_{c, i j}\left(I_{i j}\right)$.

Temperatures of $p$ - $n$ junction, of spreading current regions and ohmic contacts for each chip are assumed to be almost identical, then:

$T_{i j}=R_{t, i j} P_{i j}=R_{t, i j} V_{j} I_{i j}$,

here $R_{t, i j}$ is the junction-to-heatsink thermal resistance for an exact chip number $i$ in a section $j$.

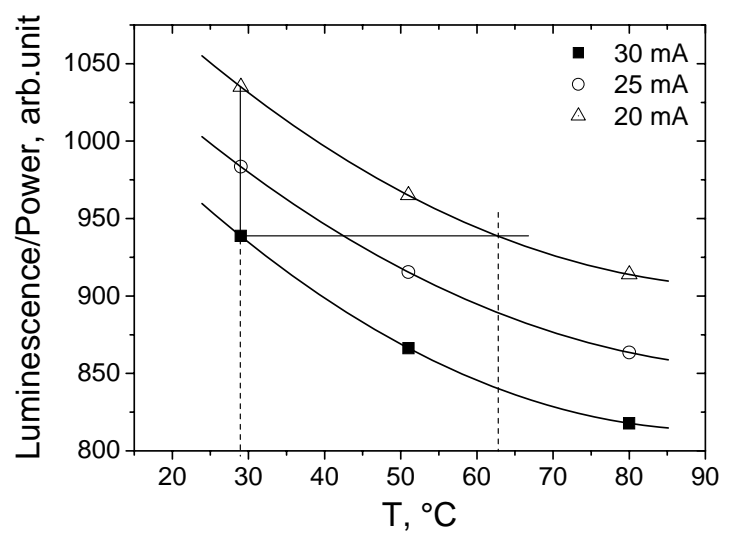

Fig. 6. The temperature dependence of the LED module efficiency at different stabilized currents.

To find the current through the chip, we write the Shockley equation:

$I_{i j}=I_{01, i j}\left(\exp \left(q V_{p n, i j} / n_{1 i j} k T_{i j}\right)-1\right)+$

$+I_{02, i j}\left(\exp \left(q V_{p n, i j} / n_{2 i j} k T_{i j}\right)-1\right)+V_{p n, i j} / R_{\text {shunt }}$,

where $I_{01, i j}=I_{001, i j} \exp \left(E_{b 1} / k T\right)$ is the saturation current, $R_{\text {shunt }}$ - shunt resistance, caused by defects.

The voltage drop on a series resistance $V_{R, i j}=I_{i j} R_{b, i j}$.

The voltage drop on the ohmic contact with the tunneling current flow:

$V_{c}=\ln \left(I_{i j} / I_{0 F, i j}\right)^{E_{00, i j}} / q$.
The current through the section $I_{j}=\sum_{i=1}^{u} I_{i j} \equiv I$ must be the same for all the module sections. The resulting voltage on the module $V=\sum_{j=1}^{w} V_{j}$.

$I-V$ characteristic of LED module is calculated in the following way: setting the current through the matrix and temperatures of chip equal to the heat sink temperature, optimizing the voltage on each section by the Newton method and reaches the given total current. The matrix voltage is calculated as amount of voltages on each section. Then, we set the temperature of each chip equal to the result of multiplication power dissipation by the chip thermal resistance. To achieve the necessary accuracy of calculation, this iterative process is repeated.

Shown in Fig. 7 is a good agreement between the calculated $I-V$ curves (solid lines), which were obtained from our model mentioned above, and experimental data (points) of LED chip. We looked for a source of $I-V$ LED module degradation and proceeded from the assumption that the main cause of degradation is single. In this case, we can explain both the increase of effective barrier height and increase of effective series resistance of module with temperature only by changing the ohmic contact parameters. It is shown in Fig. 8 that changes in values of the series resistance $R_{b}(11)$ and in values of the saturation current $I_{0 \mathrm{~F}}(14)$ may fully explain the observed changes of integrated $I-V$ characteristic. The increase of serial resistance is, probably, due to diffusion processes in ohmic contacts under the influence of heating by dissipated power. On the one hand, this process decreases the efficiency of LED module and increases the dissipation power. On the other hand, there is an adjustment of LED chips by series resistance. The series resistance of more loaded (due to variation of parameters) LED chip is increased, which results in the decreased value of current through it. This effect can be the reason for rising the LED module barrier height.

Mean and variance parameters of saturation current, of ideality factor, of heat resistance and of series resistance components were studied experimentally.

Experimental testing the LED module reliability was performed using the current $300 \mathrm{~mA}$ and at continuous loading for 9060 hours. To calculate the effective activation energy of degradation process, we divided this time interval by two regions: the first corresponded to loading for 6800 hours with the stabilized temperature $37^{\circ} \mathrm{C}$ on heat sink, and the second one - for 2260 hours with the stabilized temperature $50{ }^{\circ} \mathrm{C}$ on heat sink. Total degradation of the integrated luminous flux of LED module was $10.7 \%$. The time dependence of LED module luminous flux at the current load $300 \mathrm{~mA}$ and different temperatures of heat sink is shown in Fig. 9. 


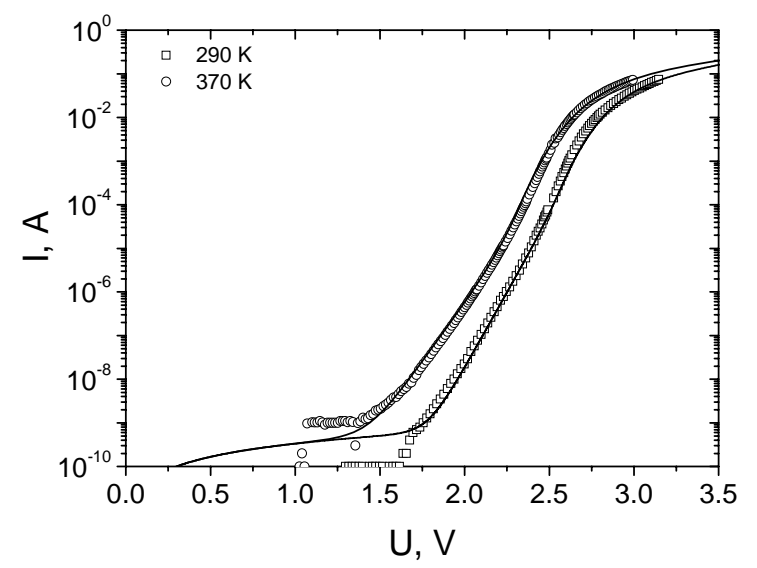

Fig. 7. $I-V$ characteristics of LED chip at different heat sink temperature $(290 \ldots 370 \mathrm{~K})$. Points - experimental data, solid lines - the result of approximation by (9), (14).

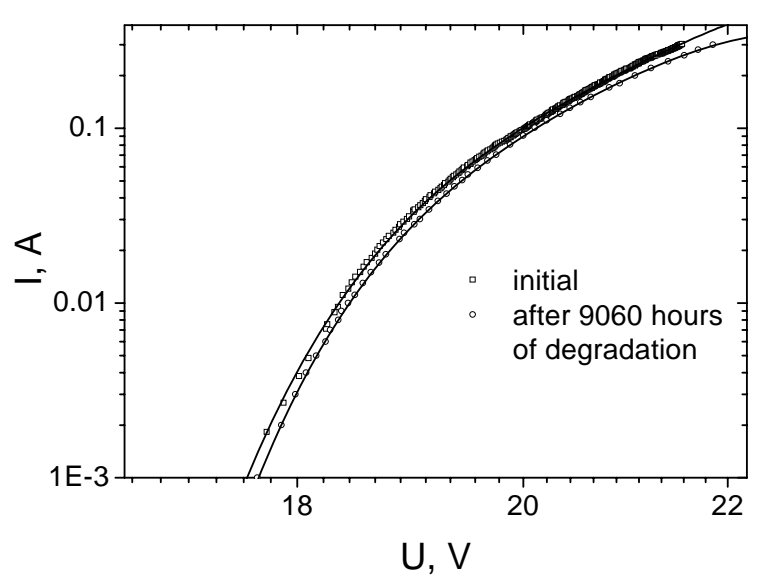

Fig. 8. Experimental $I-V$ data (points) and calculated from our model (solid lines) of LED module. Upper curve - before degradation, lower curve - after continuous load for 9060 hours.

It is easy to see that decay in the luminescence intensity is well described by an exponential function [3]:

$$
I=I_{0}[\exp (-\alpha(T) t)]
$$

where $I$ is the integrated luminescence intensity; $I_{0}$ initial integrated luminescence intensity taken after preoperation time region; $t$ - time of loading, $\alpha(T)-$ degradation coefficient that depends on temperature.

The temperature $T$ of $p-n$ junction bound with the temperature of heat sink $T_{h s}$ can be expressed as [4]:

$T=R_{T}\left[P(1-\eta(T))+I^{2} R_{s}\right]+T_{h s}$,

where $P$ is the total dissipation power in the $p-n$ junction; $\eta(T)$ - energy conversion efficiency; $R_{T}-$ junction-to-heatsink thermal resistance; $R_{S}-$ series resistance, connected with ohmic contacts series resistance and resistance of spreading current in $p^{+}$and base regions; $T_{h s}-$ heat sink temperature.
Since diffusion processes have typically activation character, the degradation coefficient can be written as [3]:

$$
\alpha(T)=\alpha_{0} \exp \left(-E_{a} / k T\right),
$$

here $E_{a}$ is the activation energy of degradation process; $k$ - Boltzmann constant; $\alpha_{0}-$ constant.

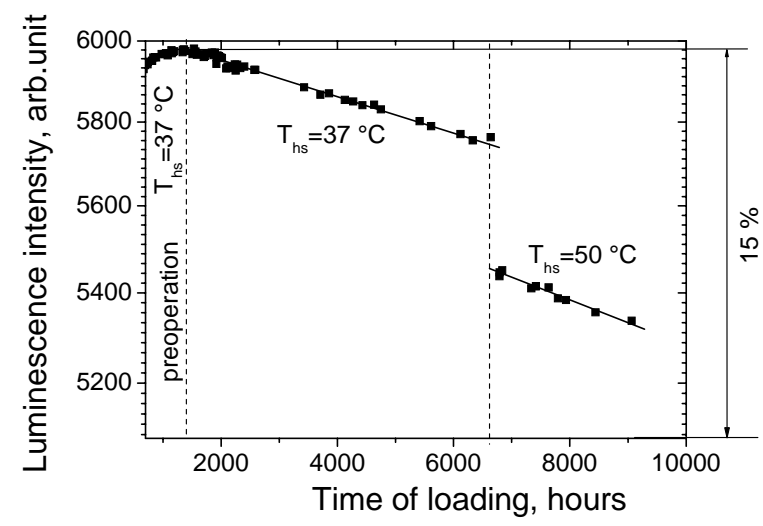

Fig. 9. Time dependence of the integrated luminescence intensity at the $300-\mathrm{mA}$ current load and stabilized temperature of heat sink.

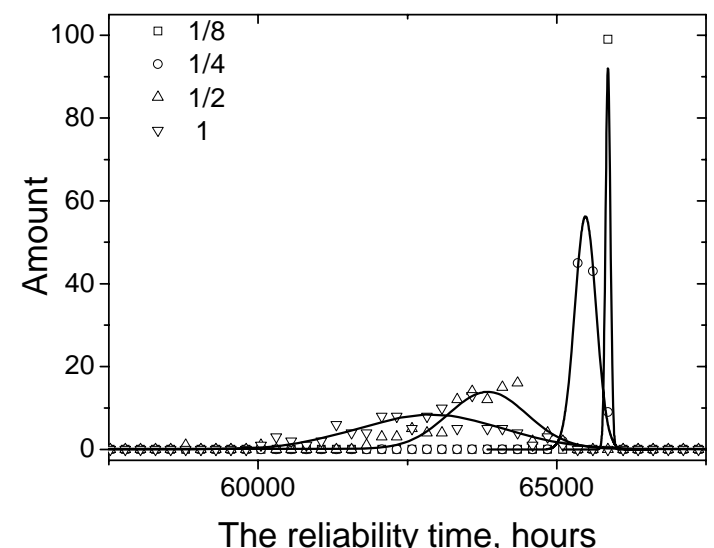

Fig. 10. The reliability dependence of LED module for different variance of LED chip resistance.

We used the Monte-Carlo method and simulate the influence of LED chips parameters variance (at the similar mean) on the rate of degradation of total LED module. Of course, we took into account two additional factors: temperature dependence of LED chip energy conversion efficiency and temperature dependence of luminescence degradation. If the variation of LED chips parameters becomes considerable, then in the LED module some LED chips with lower resistances are heated quicker, and their efficiency degrades quicker. As result, their luminescence decreases, too. Degradation of LED chip luminous flux is described by the Arrhenius equation:

\section{(C) 2016, V. Lashkaryov Institute of Semiconductor Physics, National Academy of Sciences of Ukraine}


$J_{i j}=J_{0 i j}\left(T_{i j}\right)\left[\exp \left(-\alpha_{i j}\left(T_{i j}\right) t\right)\right]$,

where: $\quad \alpha_{i j}(T)=\alpha_{0} \exp \left(-E_{a} / k T_{i j}\right)$ is the degradation coefficient; $t$ is the time of LED chip degradation; $J_{0 i j}\left(T_{i j}\right)$ - multiplier that takes into account the temperature dependence of LED chip energy conversion efficiency, which is calculated numerically being based in Fig. 3.

The reliability time was defined as the time at which LED module degradation of integrated luminescence intensity becomes $30 \%$ [5].

The result of simulating the reliability time by sampling 100 LED modules (Fig. 10) shows that the increase in the spread of series resistance of separate LED chips results in reducing the mean of reliability time and in increasing the halfwidth of reliability time for LED modules group. The curve 1 corresponds to the experimentally measured values (both for mean and variance) of series resistance. The curve $1 / 2$ corresponds to half of the variance (in the case of pre-sorting LED chip on two groups, and using only one of them for each LED module). It is easy to see that quarter of the variance (1/4) results in the essential increase of reliability time. If the spread of LED chips will be separated by 8 groups $(1 / 8)$, then the reliability time will increase from 60000 hours up to 65000 hours.

\section{Conclusions}

Being based on researches of $I-V$ characteristics, it has been shown that degradation processes in LED chip are caused by diffusion processes in ohmic contacts under the influence of dissipation power heating. As a result, degradation of the integrated luminescence intensity of LED module can be described by the activation type exponential function. The Monte-Carlo method was used to calculate the reliability time of LED modules with different halfwidth of the variance of LED chip series resistance. The reliability time of LED modules [5] was defined as the longest time at which $95 \%$ of modules will degrade to no more than $30 \%$ of the initial luminous flux. It is easy to see that, even at such soft mode of use, the reliability time of LED modules increases by 10 percent. Thus, the reliability time of LED modules may be increased by 10 percent, if using LED chips binning by 8 groups (or more) before manufacturing the module.
References

1. Solid State Lighting Reliability: Components to Systems. Ed. by W.D. van Driel, Xuejun Fan. Springer Science \& Business Media, 2012. (DOI: 10.1007/978-1-4614-3067-4).

2. Chen Zhaohui, Zhang Qin, Wang Kai, Luo Xiaobing, Liu Sheng, Reliability test and failure analysis of high power LED packages // $J$. Semiconductors, 32, No. 1, p. 014007 (1-4), 2011 (DOI: 10.1088/1674-4926/32/1/014007).

3. Physics of Semiconductor Devices, 3-rd Edition, ed. by Simon M. Sze, Kwok K. Ng. John Wiley \& Sons, 2006.

4. Light-Emitting Diodes, 2-nd Edition, ed. by E. Fred Schubert. Cambridge University Press, 2006.

5. IEC/PAS 62717:2011 LED modules for general lighting - Performance requirements (IDT). International Standard, 2014 (e-link: https://webstore.iec.ch/publication/7393). 